\title{
1 Effect of sinusoidal electrical cortical stimulation on brain cells
}

3 Seungjun Ryu ${ }^{\mathrm{a} \dagger}$, Kyung-Tai Kim ${ }^{\mathrm{b} \dagger}$, Hyeon $\mathrm{Seo}^{\mathrm{c} \dagger}$, Jongwook Cho ${ }^{\mathrm{a}}$, Jiyoung Park ${ }^{\mathrm{a}}$, Sung Chan Jun ${ }^{\mathrm{d}}$,

4 Hyoung-Ihl Kim ${ }^{\mathrm{a}}$

5

$6 \quad{ }^{a}$ Department of Biomedical and Science and Engineering, Institute of Integrated Technology,

7 Gwangju Institute of Science and Technology, Gwangju 61005, Korea

8 bJeonbuk Department of Inhalation Research, Korea Institute of Toxicology, 30 Baekhak1-gil,

9 Jeongeup, Jeollabuk-do 56212, Republic of Korea

$10{ }^{\mathrm{c}}$ Medical Device Development Center, Daegu-Gyeongbuk Medical Innovation Foundation, 41061,

11 Daegu, Korea

$12{ }^{\mathrm{d}}$ School of Electrical Engineering and Computer Science, Gwangju Institute of Science and

13 Technology, Gwangju 61005, Korea

15 †These authors have contributed equally to this work

16 Correspondence: Hyoung-Ihl Kim

17 Department of Biomedical and Science and Engineering, Institute of Integrated Technology, Gwangju

18 Institute of Science and Technology, Gwangju 61005, Korea

19 Tel:82-62-715-5302

20 Fax:82-62-715-5309

21 E-mail: hyoungihl@gist.ac.kr

22

23 Email addresses for coauthors:

24 Seungjun Ryu: perao123@gist.ac.kr

25 Kyung-Tai Kim: kyungtai.kim@kitox.re.kr

26 Hyeon Seo: hseo0612@gmail.com 
27 Jongwook Cho: chojw@ gist.ac.kr

28 Jiyoung Park: mimi73@gist.ac.kr

29 Sung Chan Jun: scjun@ gist.ac.kr

30

31 Abbreviations list

32 AP, action potential; CaMKII, calmodulin-dependent protein kinase II; CSF, cerebrospinal fluid; EBS,

33 electrical brain stimulation; PV, parvalbumin; tDCS, transcranial direct current stimulation 


\section{ABSTRACT}

36 Background: Electrical cortical stimulation is often used in patients with neurological disorders but it

37 is unclear how it modulates different types of brain cells.

38 Objective: The aim of this study was to determine the effect of sinusoidal electrical brain stimulation

39 (SEBS) on different types of brain cells and to identify the exact types of brain cells that are

40 stimulated.

41 Methods: The study subjects were 40 male Sprague Dawley rats (weight 300-350 g; age 9 weeks).

42 SEBS was delivered continuously at frequencies of $20,40,60$, or $100 \mathrm{~Hz}$ to the sensory parietal

43 cortex using epidurally placed electrodes for 1 week. Transverse rat brain tissue sections were

44 immunolabeled with calmodulin-dependent protein kinase II and parvalbumin (PV) antibodies and

45 with c-Fos for counting of activated excitatory and inhibitory neurons. Computer simulation was

46 performed to cross-validate the frequency-specific cell stimulation results.

47 Results: Inhibitory neurons were more excited than excitatory neurons after epidural EBS. Most

48 excitatory neural activity was evoked at $40 \mathrm{~Hz}(p<0.05)$ and most inhibitory neuronal activity was

49 evoked at $20 \mathrm{~Hz}(p<0.01)$. The contralateral sensory cortex was activated significantly more at $40 \mathrm{~Hz}$

$50(p<0.05)$ and the corticothalamic circuit at $20 \mathrm{~Hz}(p<0.001)$. Stimulation-induced excitatory and

51 inhibitory neuronal activation was widest at $20 \mathrm{~Hz}$.

52 Conclusions: Epidural electrical stimulation targets both excitatory and inhibitory neurons and the

53 related neural circuits. Further exploration is needed to identify circuits that promote the plasticity

54 needed for recovery in patients with specific neurological diseases.

55

56 Keywords: brain stimulation, sine waveform, inhibitory neuron, excitatory neuron, biocomputation 


\section{INTRODUCTION}

59 Electrical brain stimulation (EBS) is a type of electrotherapy that modulates neuronal activity using a

60 controlled electric current and is increasingly used alone or in combination with other clinical therapy

61 for various neurological disorders, such as essential tremor [1, 2], epilepsy [3, 4], Parkinson's disease

$62[5,6]$, chronic pain [7], depression [8], cerebral infarction, and other brain disorders [9, 10].

63 Essentially, EBS generates an electrical field that affects specific populations of neurons. Numerous

64 researchers have attempted to determine how this electrical field influences neural activity in local

65 and remote neural circuits and the casual relationship with the resulting behavioral changes. However,

66 there are many different types of neuronal cells that are under the influence of specific

67 neurotransmitters, and it is still unclear exactly how EBS works.

68 The specific function triggered when a neural circuit is stimulated depends on the type of brain cell

69 involved. Each type of neuron has a specific modulatory effect, such as post-synaptic excitation or

70 inhibition, and the different types of neuron are interconnected. Several studies have shown that the

71 efficacy of transmission at the synapse can undergo a short-term increase (known as facilitation) or

72 decrease (depression) according to the activity of the presynaptic neuron [11-14]. However, previous

73 studies of the mechanism of EBS have usually focused on the function of pyramidal neurons [15-18],

74 given that they are assumed to play a key role in activation of neural activity and the associated

75 plasticity in the stimulated cortex. Although recent studies have shown that excitatory neurons are

76 strongly regulated by inhibitory neurons via feed-forward and feedback mechanisms [19, 20], the type

77 of cells most influenced by EBS is still unknown [21-23].

78 The basic mechanisms underlying EBS include functional reorganization of neural structures,

79 substrates, and increased synaptic plasticity, which are modified by various factors, such as the

80 stimulation type and parameter, and current brain status [24-26]. Recently, transcranial alternating

81 current stimulation(tACS) has become popular because it shows entrainment of brain oscillations in a

82 frequency-specific manner and can be administered using various parameters, including sinusoidal 
83 weak intensity stimulation [23], but there is doubt regarding their effectiveness [27]. And also, there

84 remain many uncertainties regarding the interaction between neural excitability and strong sinusoidal

85 stimulation.

86 The aim of this study was to identify the neural cell populations that are activated during SEBS.

87 Computational models were incorporated to clarify the effect of SEBS on the relationship between the

88 spatial distribution of a stimulus-induced electrical field and activation of individual neurons and how

89 it alters neuronal spiking.

90

91 MATERIAL AND METHODS

92 Experimental animals

93 Forty male Sprague Dawley rats (300-350 g, aged 9 weeks) were used in the study. All experiments

94 were performed in accordance with the ARRIVE guidelines and the institutional guidelines of the

95 Gwangju Institute of Science and Technology (GIST). All procedures were approved by the

96 Institutional Animal Care and Use Committee at GIST. The rats were divided into four experimental

97 groups (to receive SEBS at 20,40,60, or $100 \mathrm{~Hz}$ ) and a sham operation group. At least 5 rats were

98 included in each study group.

\section{Surgical procedures}

100 The rats were anesthetized using a mixture of ketamine hydrochloride $100 \mathrm{mg} / \mathrm{kg}$ and xylazine 7

$101 \mathrm{mg} / \mathrm{kg}$. After 15 minutes, the rats were fixed in a small-animal stereotactic frame. Body temperature

102 was maintained at $37.5 \pm 5^{\circ} \mathrm{C}$ with a thermocouple blanket. With bregma (B) and lambda (L) in a flat

103 plane as reference points, a small craniectomy was performed $3 \mathrm{~mm}$ posterior to bregma and $3 \mathrm{~mm}$

104 lateral to the midline. All 40 rats underwent insertion of a custom-made electrode (diameter $3 \mathrm{~mm}$,

105 height $0.37 \mathrm{~mm}$ ) via craniotomy in the epidural area, with a 0.7 -mm-diameter reference screw

106 electrode placed $2 \mathrm{~mm}$ anterior to bregma and $3 \mathrm{~mm}$ lateral to the midline. The electrode extended

107 from $1.5 \mathrm{~mm}$ to $4.5 \mathrm{~mm}$ posteriorly and $1.5 \mathrm{~mm}$ to $4.5 \mathrm{~mm}$ lateral to bregma $\left(9 \mathrm{~mm}^{2}\right)$, covering the 
108 hindlimb, trunk, and forelimb areas of the sensory cortex. The electrodes were connected to a pedestal

109 on the skull, fixed and sealed with bone cement, and then connected to a stimulator (Cybermedic Co.

110 Ltd., Iksan, Korea) via a swivel adaptor at the top of the cage.

\section{Electrical stimulation}

112 Voltage stimulation was delivered continuously ( $24 \mathrm{~h} /$ day) to the sensory cortex via a programmable

113 Cybermedic stimulator for 1 week. We maintained the experimental stimulation intensity at half of the

114 individual movement threshold. On alternate days, we measured the individual motor threshold during

115 stimulation and regulated the voltage. The experimental stimulation intensities ranged from $1.0 \mathrm{~V}$ to

$1163.0 \mathrm{~V}$ and frequencies of $20,40,60$, and $100 \mathrm{~Hz}$ were used to investigate differential stimulation of

117 neuronal cells. A continuous sinusoidal waveform with a duty cycle of $99 \%$ was maintained for all

118 animals in each of the experimental groups (Fig. 1A).

\section{Neurohistological analysis}

120 Immunohistochemistry

121 All rats in each group were euthanized and processed for c-Fos immunohistochemistry after 1 week of

122 cortical stimulation. Immunohistochemistry was performed as described previously [28]. Briefly, rats

123 were perfused with 4\% paraformaldehyde (PFA), post-fixed in 4\% PFA overnight, and the brains were

124 sunk to $30 \%$ sucrose for cryoprotection. Coronal brain sections $(40 \mu \mathrm{m})$ were performed using

125 microtome. Rat brain sections were incubated with following antibodies: rabbit anti-c-Fos (1:1000)

126 (Cell Signaling, 2250S), Mouse anti-CamKII (Abcam, ab22609), Guinea pig anti-parvalbumin

127 (Synaptic systems, 195 004), goat anti-guinea pig alexa 555 (1:200) (Invitrogen, A21435). Proper

128 fluorophore-conjugated secondary antibody (Invitrogen) was used and images were captured using

129 LSM-800 confocal microscope (Zeiss).

130 For DAB staining, the brain sections were treated with $3 \% \mathrm{H}_{2} \mathrm{O}_{2}$ in Tris-buffered saline and $1 \%$

131 normal goat serum and then incubated in c-Fos 9F6 rabbit antibody (Cell Signaling Technology). The

132 sections were incubated in a Polink-1 horseradish peroxidase detection system for rabbit antibody 
133 (GBI Labs, Mukilteo, WA, USA) on the following day. After a color reaction was observed on

134 incubating sections with diaminobenzidine/peroxidase solution (DAB $0.02 \% ; 0.08 \%$ nickel sulfate) in

135 Tris-buffered saline, the brain sections were mounted on gelatin-coated slides. c-Fos images were

136 captured using a Leica microscope.

137 c-Fos mapping

138 Fast Fourier transform-bandpass filtered images were created in ImageJ (National Institutes of Health,

139 Bethesda, MD, USA) and cell density maps using a custom MATLAB-based program (MathWorks,

140 Natick, MA, USA) [29]. A sample image showing the results of the transfer function when applied is

141 shown in Fig. 1B. Regions of interest were selected on the motor and sensory cortices, striatum, and

142 thalamus, and the number of c-Fos-positive cells in each region of interest was counted automatically

143 by calculating the mean image pixel intensity and applying a threshold, with validation by

144 microscopic counting.

145 Quantification

146 To quantify activated excitatory or inhibitory neurons in rat cortex, $40 \mu \mathrm{m}$-thick transverse sections of

147 were immunolabeled with c-Fos, PV, CamKII antibodies and counted the number of double positive

148 cells (activated excitatory neurons: c-Fos and CamKII, activated inhibitory neurons: c-Fos and PV).

149 At least 4 brains were analyzed for each group. All quantifications in images were analyzed in ImageJ

150 software.

\section{Statistical analysis}

152 The study data were analyzed using OriginPro version 9.1 software (OriginLab, Northampton, MA,

153 USA). The data were assessed for normality using the Kolmogorov-Smirnov test. The numbers of

154 cells expressing c-Fos were then compared between the study groups using one-way analyses of

155 variance. The Bonferroni post-hoc test was used to detect significant differences between groups for

156 each region of interest and specific cell type. If no significant differences were detected, Kruskal-

157 Wallis one-way analysis of variance by ranks with Dunn's method was used to compare the specific 
158 numbers of cells co-labeled with c-Fos between the study groups and for post hoc comparison. A $p$ -

159 value $<0.05$ was considered statistically significant.

\section{Computational simulation}

161 Stimulus-induced potential field

162 We constructed a three-dimensional finite element model of a rat head using computed tomography

163 images of a rat. The rat brain imaging was performed using a volumetric micro-CT scanner (NFR

164 Polaris G90C; NanoFocusRay, Ikson, Korea). The image size was 1024×1024 pixels with 434 slices

165 and the voxel size was $0.0698 \times 0.0698 \times 0.1396 \mathrm{~mm}^{3}$. Manual segmentation was performed using

166 Seg3d to guarantee continuity and to improve the accuracy of segmentation. The model consisted of

167 the scalp, skull, and cerebrospinal fluid (CSF); we included the brain after shrinking the CSF layer by

$1685 \mathrm{~mm}$. The electrodes were modeled in accordance with the surgical procedures. The rat head model

169 was generated by an optimized tetrahedral mesh using Iso2Mesh toolbox [30], TetGen [31], and

170 MATLAB.

171 The electrical properties of each tissue, taken from averaged human conductivity values, were

172 assigned as follows (S/m): skin, 0.45; skull, 0.01; CSF, 1.65; brain, 0.2; and electrode, 5.5e07. The

173 potential field were calculated by solving the quasi-static Laplace equation via COMSOL

174 Multiphysics (v5.3, COMSOL. Inc., Burlington, MA, USA) using the finite element method. We

175 applied the conjugate gradient method with preconditioning of an algebraic multigrid (relative

176 tolerance, $1 \times 10^{-6}$; Fig. 2)

177 Neuronal responses to the electrical field

178 Single-compartment models with Hodgkin-Huxley properties were modified to represent regular

179 spiking excitatory neurons and fast spiking interneurons [22]. The constants and parameters were

180 unchanged from the original models, which resulted in different firing patterns with respect to various

181 frequencies and the strength of sinusoidal stimulation. The neuronal models were implemented in

182 NEURON [32]. 
183 To simulate neuronal responses according to the predicted potential distributions calculated in the rat

184 head model, we constructed a multi-scale model that virtually combined the single-compartment

185 neuronal models with the rat head model $[33,34]$. The multi-scale model consisted of the following

186 two-step process. First, the excitatory and inhibitory neurons were distributed on the cortical surface.

187 Second, the external stimulus calculated using the rat head model was applied to neuronal models.

188 Therefore, the external input $\mathrm{I}(\mathrm{t})=\mathrm{I}_{0} f(t)$ was added to the cable model where $\mathrm{I}_{0}=\partial^{2} \mathrm{~V} / \partial \mathrm{s}^{2}$

189 approximates the amplitude of the stimulus-induced transmembrane current and $f(t)$ represents the

190 pulse waveform. The amplitude of the extra current $I_{0}$ is determined by an "activating function" that

191 evokes activation of neuronal models [35]. S is the direction that is locally parallel with the fiber, and

192 we assumed the fiber direction to be the normal direction of the close element comprising the cortical

193 surface. The $I_{0}$ was calculated at each neuron's position in the rat head model using COMSOL with

194 MATLAB. For the waveform, we simulated continuous sinusoidal stimulation at 20, 40, 60, 80, and

$195100 \mathrm{~Hz}$ in accordance with the animal experiment.

\section{RESULTS}

\section{Quantification of neuronal activity from expression of c-Fos after SEBS}

199 c-Fos is an immediate-early gene that responds transiently and rapidly to various stimuli and is a good

200 marker of neuronal activation in the brain [36]. To identify neuronal activation by SEBS in the rat

201 brain, we performed SEBS at various frequencies (20, 40, 60, and $100 \mathrm{~Hz}$ ) $24 \mathrm{~h} /$ day for 1 week (Fig.

202 1A). The immunoreactivity of c-Fos shows the neuronal activation density map at two different

203 bregma levels after SEBS at 20,40,60, and $100 \mathrm{~Hz}$ in the experimental groups and in the sham group

204 (Fig. 3A). Activation of c-Fos by SEBS was propagated to various brain regions including not only

205 the motor and sensory cortices but also the deeper brain, including the striatum and thalamus. To

206 elucidate the neuronal activation in the various regions, we performed automated c-Fos positive cell

207 counts in four regions of interest (motor cortex, sensory cortex, striatum, thalamus) among the groups

208 (Table S1). SEBS at $40 \mathrm{~Hz}$ resulted in the highest significant increment of c-Fos-positive cell count in 
209 the contralateral sensory cortex $(p<0.05)$. In the $20 \mathrm{~Hz}$ SEBS group, activation was highest in the

210 thalamus $(p<0.001)$. Regardless of frequency, the increments in neuronal activity were significantly

211 greater in the EBS groups than in the sham group (Fig. 3B, Table S1).

\section{Quantification of sensory cortical cell type-specific activity using co-labeling with c-Fos}

\section{4 expression after SEBS}

215 Excitatory/inhibitory balance is required for correct functioning of the brain. Because SEBS

216 modulates the excitatory/inhibitory neuronal balance, it has been applied as effective treatment for

217 various neurological disorders [37-39]. To elucidate the functional mechanism of SEBS, we

218 investigated the types of neuronal cells that are activated after SEBS. Immunohistochemistry was

219 performed with calmodulin-dependent protein kinase II (CaMKII) antibody for excitatory neurons and

220 PV antibody for inhibitory neurons. Activated inhibitory neurons $\left(\mathrm{PV}^{+}, \mathrm{c}-\mathrm{Fos}{ }^{+}\right)$and excitatory neurons

221 (CaMKII $\left.{ }^{+}, \mathrm{c}-F o s^{+}\right)$were analyzed (Fig. 4).

222 20Hz SEBS markedly improved the activity of inhibitory neurons in both cortices but not that of

223 excitatory neurons when compared with the sham group (ipsilateral side; $86.98 \pm 2.12 \%, p<0.001$;

224 contralateral side; $80.54 \pm 8.88 \%, p<0.001)$. Even though $40 \mathrm{~Hz}$ and $60 \mathrm{~Hz}$ SEBS increased the activity

225 of inhibitory neurons, excitatory neurons were more activated than those in the sham group (40 $\mathrm{Hz}$ on

226 ipsilateral side, $59.82 \pm 3.25 \%, p<0.001 ; 40 \mathrm{~Hz}$ on contralateral side, $54.22 \pm 3.29 \%, p<0.01 ; 60 \mathrm{~Hz}$ on

227 ipsilateral side, $49.54 \pm 4.70 \%, p<0.05 ; 60 \mathrm{~Hz}$ on contralateral side, 51.30 $\pm 2.26 \%, p=0.09$; Fig. 4C).

228 We also calculated the normalized ratio from the average value in the sham group to compare the

229 excitatory and inhibitory neurons at each frequency (Fig. 4D) and specific regional neurons between

230 three different frequencies (Fig. 4E). The activity of inhibitory neurons was markedly increased by

$23120 \mathrm{~Hz}$ SEBS when compared with the activity of excitatory neurons (ipsilateral side, $3.49 \pm 0.09$ and

$2321.04 \pm 0.08$, respectively, $p<0.001$; contralateral side, $2.41 \pm 0.27$ and $0.87 \pm 0.08, p<0.001)$. The activity

233 of inhibitory neurons was increased by $40 \mathrm{~Hz}$ and $60 \mathrm{~Hz}$ SEBS; however, the increase was only 
234 statistically significant on the contralateral side ( $40 \mathrm{~Hz}$ on ipsilateral side, $1.98 \pm 0.29$ and $1.87 \pm 0.10$;

$23540 \mathrm{~Hz}$ on contralateral side, $1.91 \pm 0.14$ and $1.46 \pm 0.09, p<0.05 ; 60 \mathrm{~Hz}$ on ipsilateral side, $2.03 \pm 0.19$ and

$2361.55 \pm 0.15 ; 60 \mathrm{~Hz}$ on contralateral side, $1.86 \pm 0.19$ and $1.35 \pm 0.08, p<0.05$; Fig. 4D).

237 The activity of excitatory neurons that received $40 \mathrm{~Hz}$ and $60 \mathrm{~Hz}$ SEBS was significantly greater than

238 that of excitatory neurons that received $20 \mathrm{~Hz}$ SEBS on both the ipsilateral and contralateral sides

239 ( $p<0.05$ and $p<0.05$, respectively); however, the activity of inhibitory neurons was significantly

240 greater in response to $20 \mathrm{~Hz}$ SEBS than in response to $40 \mathrm{~Hz}$ and $60 \mathrm{~Hz}$ SEBS only on the ipsilateral

$241 \quad$ side $(p<0.01 ;$ Fig. 4E)

242 CaMKII-positive excitatory neurons were predominantly located in layers $2 / 3$ and 6 but PV-positive

243 inhibitory neurons were mainly found in layers 4 and 5 [40, 41]. We quantified the number of neurons

244 activated according to the cortical layer in which they were located by dividing the cortex into 10 bins

245 (Supplementary Fig. 2A, 2B). We found that 40Hz SEBS activated excitatory neurons in the whole

246 cortical layer but that $60 \mathrm{~Hz}$ SEBS only activated neurons in layers $2 / 3$ and 6 . Although $20 \mathrm{~Hz}$ SEBS

247 did not activate excitatory neurons, it activated inhibitory neurons in the entire cortical layer, and 40-

$248 \mathrm{~Hz}$ and 60-Hz SEBS activated inhibitory neurons in the deeper layer (Supplementary Fig. 2C, 2D).

249 Computational simulation for observing the effect of SEBS on geometrical and neuronal

250 responses at different frequencies

251 We performed computational simulation to identify the geometrical impact of SEBS on activation of

252 neurons and their firing rate. First, the current density induced by a 1-V stimulus amplitude was

253 computed (Fig. 5). As expected, there was a higher current density in the CSF because of higher

254 conductivity, and the current density was strongest in the brain area directly beneath the electrode. A

255 high current density is observed at the edge of the active electrode because of the edge effect whereas

256 the reference electrode has less of an edge effect because it is smaller. Second, in order to elucidate

257 the functional mechanism of different excitatory/inhibitory neuronal activation by SEBS, we

258 simulated single neuron responses by increasing the external stimulus. The action potentials (APs) 
259 efficiency, which is the percentage of action potentials per stimulation pulse, is shown in Fig. 6, and

260 clearly shows the firing patterns. Generally, there was no firing for a lower stimulus amplitude with a

261 higher stimulus frequency and there was burst firing for a stronger stimulus amplitude with a lower

262 stimulus frequency. Following current-controlled stimulation, phase-locked firing patterns were

263 frequently observed in both inhibitory and excitatory neuron models. The stimulus amplitude needed

264 to evoke APs was lower for inhibitory neurons than for excitatory neurons. Third, we coupled

265 individual neurons to spatial patterns of a stimulus-induced current field calculated using a rat head

266 model (Fig. 7). Consistent with the spatial distributions of current density shown in Fig. 5, the neurons

267 were activated directly beneath the electrodes regardless of stimulus frequency and type of neuronal

268 model. Inhibitory neurons were more strongly activated than excitatory neurons because of intrinsic

269 differences between these two types of neurons. The stimulus amplitude needed to evoke neuronal

270 activation increased monotonically with stimulus frequency; therefore, 20-Hz SEBS induced the

271 strongest activation in both inhibitory and excitatory neurons, with shrinking of the activated area as

272 the frequency increased (Fig. 7).

\section{DISCUSSION}

275 The precise mechanism underlying neuronal activation by SEBS is not well understood. Electrical

276 stimulation of the sensory and motor cortex usually focus on selective stimulation of cortical

277 pyramidal cells because pyramidal neurons are known to be the primary activators of the corticospinal

278 tract and may provide the main input to the direct pathway [42, 43]. Based on experimental results

279 showing higher activation in inhibitory neurons $[15,44]$, the possibility of cell type-specific

280 individual neuron responses being a bridge to interpolation of neural networks has been raised.

281 To improve our understanding of this mechanism, we performed an animal experiment and

282 biocomputation. Synaptic connectivity and the strength of individual neurons are usually assessed by

283 intracellular recordings; however, it is impractical to record data for all neurons within a neural circuit.

284 As an alternative, we observed the firing properties of the neural circuit by eliciting a response in 
285 individual pyramidal cells and interneurons displaying a diverse activation pattern, reflecting their

286 anatomical structure using a computational study.

\section{Neuronal responses in vivo}

288 Our experimental study revealed expression of c-Fos, which represents neuronal activity [36], to be

289 higher in all of the SEBS groups than in the sham group. Cell type-specific analysis showed that

290 neuronal activity was stronger in PV+ interneurons, regardless of type of SEBS, than in the sham

291 group. This is consistent with a previous result for transcranial direct current stimulation (tDCS),

292 which predominantly modulates interneurons [45]. Interneurons are primarily inhibitory in the central

293 nervous system and their main role is to conduct flow of neuronal signals between a motor neuron or

294 sensory neuron in a neural circuit. PV interneurons are crucial when performing high-order functions

295 such as learning and decision-making and also regulate the activity of pyramidal neurons [46].

296 Therefore, our findings afford a clue for understanding the effect of current SEBS, such as tACS, in

297 specific neuronal diseases.

298 SEBS of the sensory parietal cortex and motor cortex enhanced neural activity locally beneath the area of the electrical field, unlike at the striatum and thalamus, which were distant from the

300 electromagnetic field used in our experiments. SEBS differentially affects the local electromagnetic

301 strength at the circuit level. Thalamic activation was greater at $20 \mathrm{~Hz}$ than at other frequencies. These

302 findings suggest that a neural circuit, e.g., the cortico-striatal-thalamic circuit of the salience network

303 or the corticothalamic circuit, will also be influenced by SEBS. This modulation of circuitry could

304 represent additional clue for therapeutic intervention.

305 The effects of EBS were divided into those that occurred during stimulation and those that occurred

306 after stimulation. Those that occur during stimulation are solely dependent on changes in the

307 membrane potential while those that occur after stimulation depend on membrane depolarization [15]

308 and synaptic modulation [47]. The aftereffects of cathodal tDCS depend on modulation of

309 glutamatergic synapses [15]. The mechanism of action of SEBS in the context of specific neurons 
310 could be different from the simple summation of anodal and cathodal tDCS effects The phenotypic

311 effect is represented as the balance of excitatory and inhibitory neuronal responses. Our simulation

312 results showed that $20 \mathrm{~Hz}$ SEBS evokes the strongest inhibitory and excitatory neuronal responses in

313 both model of rat and human brain (Fig. 7, Supplementary Fig. 1). Since the inhibitory neuronal

314 simulation responses are much larger than excitatory simulation neuronal responses in the group that

315 received $20 \mathrm{~Hz}$ SEBS, our clinical findings can be interpreted as meaning that stronger inhibitory

316 neuronal responses occurred in the group that received 20Hz SEBS (Fig. 4D) and that excitatory

317 neuronal activity occurred in the groups that received $40 \mathrm{~Hz}$ or $60 \mathrm{~Hz}$ SEBS (Fig. 4E).

\section{Individual neuronal responses in silico}

319 We simulated the activation of an inhibitory and excitatory neuron model by extracellular stimulation

320 and examined the relationship between the neuronal firing profile and stimulus frequency in respect to

321 realistic stimulus-induced field distributions. We found neuronal excitability to be reduced in response

322 to a strong stimulus frequency and inhibitory neurons were more sensitive than excitatory neurons to

323 sinusoidal stimulation.

324 We adapted an established model of excitatory and inhibitory neurons from Mahmud et al [22]. In

325 their model, an applied extracellular stimulation current was calculated by a derivative of potential

326 field, which can be interpreted by intracellular current stimulation. In our study, as an alternative, we

327 adjusted the stimulus amplitude via the activating function calculated using the simulated current field

328 in the rat head model. Therefore, we were able to take into account the neuron's location relative to

329 the electrodes, which may be an important determinant of neuronal polarization [48].

330 Additionaly we simulated the spatial distribution of the firing rate using a human head model to

331 investigate the impact of the complex geometry of the human brain on neuronal activation, as

332 depicted in Supplementary Fig. S1. We used a previously developed human head model [48, 49] and

333 coupled it to the same type of neuronal model in the rat head. As we placed the reference electrode on

334 the chest far from the active electrode for the human model, the activation of neurons was restricted to 
335 the area of the sensory cortex directly beneath the electrode. The complex patterns reflecting the

336 complex anatomy of the brain were observed to result in activations in the sulcal wall. As expected, a

337 20-Hz sinusoidal stimulation produced the largest areas of activation and the stimulus threshold was

338 lower for inhibitory neurons than for excitatory neurons (Fig. 7 and Supplementary Fig. S1).

339 Most computational approaches have presented neuronal excitability with sinusoidal stimulation

340 subject to a uniform electric field. Aspart et al reported a frequency-dependent polarization profile

341 using a biophysically detailed model of pyramidal neurons in response to a weak uniform electric

342 field [50]. Yi et al suggested that the geometric features of a neuron model play a crucial role in

343 determining the polarization when using a two-compartment neuron model with a uniform electric

344 field [51]. Thus, in the future, we need to achieve morphological features of neuronal models for

345 precise simulation; however, while most simulation studies investigating neuronal activation involve

346 intracellular or extracellular stimulation with a uniform electric field, we propose a multi-scale model

347 and thus we could consider extracellular field induced in the brain by the stimulating electrodes.

348 This study has some limitations. First, c-Fos activation reflects rapid responses after SEBS. In this

349 study, SEBS was performed for 1 week, so measurement of c-Fos cannot fully represent the neuronal

350 responses that occur with long-term SEBS. However, 1 week of SEBS can modulate synapses at the

351 neuronal circuit level and we assume that our c-Fos density mapping shows the chronic effects of

352 SEBS. Second, the translation of individual neuronal responses into oscillations at the network level is

353 not trivial. However, modulation of individual neurons may provide evidence of entrainment of neural

354 oscillations at the network level through which neurons are recruited by sinusoidal stimulation. Future

355 studies should incorporate synaptic connections, which may allow more precise and effective

356 application of epidural EBS in both the clinical and basic research settings. 


\section{CONCLUSION}

362 We examined the change in stimulation parameters when stimulating brain cells and the quantity of

363 each component of brain cells to determine which cells are differentially influenced after delivering

364 cortical stimulation. Our findings were derived from in vivo and in silico experiments and analyzed in

365 an integrated manner. We found that $20-\mathrm{Hz}$ SEBS is the most effective frequency for selective

366 inhibitory cortical stimulation and that 40-Hz SEBS is the most effective frequency for selective

367 excitatory cortical stimulation. We assumed the mechanism involves computational simulation

368 whereby $20-\mathrm{Hz}$ SEBS differentially stimulates inhibitory neurons and inhibits excitatory neurons

369 sequentially. In order to obtain a functional effect by applying SEBS clinically, it is necessary to

370 consider the effect of SEBS on specific neurons and neuronal circuits in specific neurological

371 disorders.

\section{Acknowledgments}

373 The authors are grateful to Professor MC Lee for his enthusiastic discussion about differential cell

374 counting based on his neuropathologic knowledge.

\section{Funding}

376 This work was supported by the Basic Science Research Program through the National Research

377 Foundation of Korea funded by the Ministry of Science and ICT (NRF-2016R1A2B3009660, NRF-

378 2019M3C1B8090841, and NRF-2018R1D1A1B07041224)

\section{Declarations of interest}

380 None.

\section{$381 \quad$ Data statement}

382 The datasets generated and analyzed during the current study are available from the corresponding

383 author on reasonable request. 
390 [1] Benabid AL, Pollak P, Hoffmann D, Gervason C, Hommel M, Perret J, et al. Long-

391 term suppression of tremor by chronic stimulation of the ventral intermediate thalamic

392 nucleus. The Lancet 1991;337(8738):403-6.

393 [2] Hubble J, Busenbark K, Wilkinson S, Penn R, Lyons K, Koller W. Deep brain

394 stimulation for essential tremor. Neurology 1996;46(4):1150-3.

395 [3] Fisher RS, Velasco AL. Electrical brain stimulation for epilepsy. Nature Reviews

396 Neurology 2014;10(5):261.

397 [4] Fish D, Gloor P, Quesney F, Oliver A. Clinical responses to electrical brain

398 stimulation of the temporal and frontal lobes in patients with epilepsy: pathophysiological

399 implications. Brain 1993;116(2):397-414.

400 [5] Group D-BSfPsDS. Deep-brain stimulation of the subthalamic nucleus or the pars

401 interna of the globus pallidus in Parkinson's disease. N Engl J Med 2001;345(13):956-63.

402 [6] Benabid AL. Deep brain stimulation for Parkinson's disease. Curr Opin Neurobiol 403 2003;13(6):696-706.

404 [7] O'connell NE, Marston L, Spencer S, DeSouza LH, Wand BM. Non $\square$ invasive brain

405 stimulation techniques for chronic pain. Cochrane Database Syst Rev 2018(3).

406 [8] Mayberg HS, Lozano AM, Voon V, McNeely HE, Seminowicz D, Hamani C, et al.

407 Deep brain stimulation for treatment-resistant depression. Neuron 2005;45(5):651-60.

408 [9] Kim RG, Cho J, Ree J, Kim H-S, Rosa-Neto P, Kim J-M, et al. Sensory-parietal

409 cortical stimulation improves motor recovery in severe capsular infarct. J Cereb Blood Flow

410 Metab 2016;36(12):2211-22.

411 [10] Webster BR, Celnik PA, Cohen LG. Noninvasive brain stimulation in stroke

412 rehabilitation. NeuroRx 2006;3(4):474-81.

413 [11] Regehr WG. Short-term presynaptic plasticity. Cold Spring Harb Perspect Biol 414 2012;4(7):a005702.

415 [12] Nadim F, Manor Y. The role of short-term synaptic dynamics in motor control. Curr 416 Opin Neurobiol 2000;10(6):683-90.

417 [13] Dittman JS, Kreitzer AC, Regehr WG. Interplay between facilitation, depression, and 418 residual calcium at three presynaptic terminals. J Neurosci 2000;20(4):1374-85.

419 [14] Jackman SL, Regehr WG. The mechanisms and functions of synaptic facilitation. 420 Neuron 2017;94(3):447-64.

421 [15] Nitsche M, Fricke K, Henschke U, Schlitterlau A, Liebetanz D, Lang N, et al. 422 Pharmacological modulation of cortical excitability shifts induced by transcranial direct 423 current stimulation in humans. The Journal of physiology 2003;553(1):293-301.

424 [16] Schulz R, Gerloff C, Hummel FC. Non-invasive brain stimulation in neurological 
425 diseases. Neuropharmacology 2013;64:579-87.

426 [17] Delvendahl I, Gattinger N, Berger T, Gleich B, Siebner HR, Mall V. The role of pulse

427 shape in motor cortex transcranial magnetic stimulation using full-sine stimuli. PLoS One

$428 \quad 2014 ; 9(12): \mathrm{e} 115247$.

429 [18] Kim Y-H, You SH, Ko M-H, Park J-W, Lee KH, Jang SH, et al. Repetitive

430 transcranial magnetic stimulation-induced corticomotor excitability and associated motor

431 skill acquisition in chronic stroke. Stroke 2006;37(6):1471-6.

432 [19] Maffei A, Nelson SB, Turrigiano GG. Selective reconfiguration of layer 4 visual

433 cortical circuitry by visual deprivation. Nat Neurosci 2004;7(12):1353.

434 [20] Bannister AP. Inter-and intra-laminar connections of pyramidal cells in the neocortex.

435 Neurosci Res 2005;53(2):95-103.

436 [21] Antal A, Paulus W. Transcranial alternating current stimulation (tACS). Front Hum

437 Neurosci 2013;7:317.

438 [22] Mahmud M, Vassanelli S. Differential Modulation of Excitatory and Inhibitory

439 Neurons during Periodic Stimulation. Front Neurosci 2016;10:62.

440 [23] Tavakoli AV, Yun K. Transcranial Alternating Current Stimulation (tACS)

441 Mechanisms and Protocols. Front Cell Neurosci 2017;11:214.

442 [24] Ridding M, Ziemann U. Determinants of the induction of cortical plasticity by

443 non $\square$ invasive brain stimulation in healthy subjects. The Journal of physiology

444 2010;588(13):2291-304.

445 [25] Nudo RJ. Functional and structural plasticity in motor cortex: implications for stroke

446 recovery. Physical Medicine and Rehabilitation Clinics 2003;14(1):S57-S76.

447 [26] Khatoun A, Asamoah B, Mc Laughlin M. Simultaneously excitatory and inhibitory

448 effects of transcranial alternating current stimulation revealed using selective pulse-train

449 stimulation in the rat motor cortex. J Neurosci 2017:1390-17.

450 [27] Asamoah B, Khatoun A, Mc Laughlin M. tACS motor system effects can be caused

451 by transcutaneous stimulation of peripheral nerves. Nature communications 2019;10(1):266.

452 [28] Kim K-T, Kim N, Kim H-K, Lee H, Gruner HN, Gergics P, et al. ISL1-based LIM

453 complexes control Slit2 transcription in developing cranial motor neurons. Sci Rep

454 2016;6:36491.

455 [29] Wada M, Yoshimi K, Higo N, Ren Y-R, Mochizuki H, Mizuno Y, et al. Statistical

456 parametric mapping of immunopositive cell density. Neurosci Res 2006;56(1):96-102.

457 [30] Fang Q, Boas DA. Tetrahedral mesh generation from volumetric binary and

458 grayscale images. 2009 IEEE International Symposium on Biomedical Imaging: From Nano

459 to Macro. Ieee; 2009:1142-5.

460 [31] Si H, Gärtner K. Meshing piecewise linear complexes by constrained Delaunay

461 tetrahedralizations. Proceedings of the 14th international meshing roundtable. Springer;

462 2005:147-63.

463 [32] Hines ML, Carnevale NT. The NEURON simulation environment. Neural Comput

464 1997;9(6):1179-209.

465 [33] Seo H, Jun SC. Multi-Scale Computational Models for Electrical Brain Stimulation.

466 Front Hum Neurosci 2017;11:515.

467 [34] Seo H, Jun SC. Relation between the electric field and activation of cortical neurons

468 in transcranial electrical stimulation. Brain Stimul 2019;12(2):275-89.

469 [35] Rattay F. Analysis of models for external stimulation of axons. IEEE Trans Biomed

$470 \quad$ Eng 1986(10):974-7.

471 [36] Bullitt E. Expression of $\mathrm{c} \square$ fos $\square$ like protein as a marker for neuronal activity 
472 following noxious stimulation in the rat. J Comp Neurol 1990;296(4):517-30.

473 [37] De Ridder D, De Mulder G, Menovsky T, Sunaert S, Kovacs S. Electrical stimulation 474 of auditory and somatosensory cortices for treatment of tinnitus and pain. Prog Brain Res

$475 \quad 2007 ; 166: 377-88$.

476 [38] Morrell MJ. Responsive cortical stimulation for the treatment of medically

477 intractable partial epilepsy. Neurology 2011;77(13):1295-304.

478 [39] Treister R, Lang M, Klein MM, Oaklander AL. Non-invasive transcranial magnetic

479 stimulation (TMS) of the motor cortex for neuropathic pain-at the tipping point? Rambam

480 Maimonides medical journal 2013;4(4).

481 [40] Tremblay R, Lee S, Rudy B. GABAergic interneurons in the neocortex: from cellular 482 properties to circuits. Neuron 2016;91(2):260-92.

483 [41] Wang X, Zhang C, Szábo G, Sun Q-Q. Distribution of CaMKII $\alpha$ expression in the 484 brain in vivo, studied by CaMKII $\alpha$-GFP mice. Brain Res 2013;1518:9-25.

485 [42] Gorman At. Differential patterns of activation of the pyramidal system elicited by 486 surface anodal and cathodal cortical stimulation. J Neurophysiol 1966;29(4):547-64.

487 [43] Zwartjes DG, Heida T, Feirabend HK, Janssen ML, Visser-Vandewalle V, Martens

488 HC, et al. Motor cortex stimulation for Parkinson's disease: a modelling study. Journal of 489 neural engineering 2012;9(5):056005.

490 [44] Nitsche MA, Seeber A, Frommann K, Klein CC, Rochford C, Nitsche MS, et al. 491 Modulating parameters of excitability during and after transcranial direct current stimulation

492 of the human motor cortex. The Journal of physiology 2005;568(1):291-303.

493 [45] Lang N, Siebner HR, Ward NS, Lee L, Nitsche MA, Paulus W, et al. How does

494 transcranial DC stimulation of the primary motor cortex alter regional neuronal activity in the 495 human brain? Eur J Neurosci 2005;22(2):495-504.

496 [46] English DF, McKenzie S, Evans T, Kim K, Yoon E, Buzsáki G. Pyramidal cell497 interneuron circuit architecture and dynamics in hippocampal networks. Neuron

498 2017;96(2):505-20. e7.

499 [47] Braithwaite JJ, Mevorach C, Takahashi C. Stimulating the aberrant brain: Evidence

500 for increased cortical hyperexcitability from a transcranial direct current stimulation (tDCS)

501 study of individuals predisposed to anomalous perceptions. Cortex 2015;69:1-13.

502 [48] Seo H, Kim D, Jun SC. Effect of Anatomically Realistic Full-Head Model on

503 Activation of Cortical Neurons in Subdural Cortical Stimulation-A Computational Study. Sci

504 Rep 2016;6:27353.

505 [49] Seo H, Kim HI, Jun SC. The Effect of a Transcranial Channel as a Skull/Brain

506 Interface in High-Definition Transcranial Direct Current Stimulation-A Computational Study.

$507 \quad$ Sci Rep 2017;7:40612.

508 [50] Aspart F, Remme MW, Obermayer K. Differential polarization of cortical pyramidal

509 neuron dendrites through weak extracellular fields. PLoS Comput Biol 2018;14(5):e1006124.

510 [51] Yi G-S, Wang J, Wei X-L, Tsang K-M, Chan W-L, Deng B, et al. Exploring how

511 extracellular electric field modulates neuron activity through dynamical analysis of a two-

512 compartment neuron model. J Comput Neurosci 2014;36(3):383-99. 
bioRxiv preprint doi: https://doi.org/10.1101/855395; this version posted November 26,2019 . The copyright holder for this preprint (which was not certified by peer review) is the author/funder, who has granted bioRxiv a license to display the preprint in perpetuity. It is made available under aCC-BY-NC-ND 4.0 International license.

516

517 


\section{FIGURE LEGENDS}

Figure 1. Schematic diagram of the experimental protocol and an example of cell density heat

map of c-Fos-positive cells. (A) Experimental procedure for SEBS in Sprague Dawley rats. (B)

Image showing the customized transfer function for cell density maps of c-Fos-positive cells. EBS,

electrical brain stimulation

Figure 2. High-resolution computed tomography scan based on an anatomically realistic rat

526 head model. The anatomically realistic rat head model consisted of four layers of skin (A), skull (B),

527 cerebrospinal fluid (C), and brain (D). The electrodes are placed in accordance with the coordinates

528 used in the experiment (E), and a cross-section, following the black dotted line shown in (E), passing

529 the reference $(F)$ and the active electrode $(\mathrm{G})$ are shown.

531 Fig. 3. Expression of c-Fos after sensory-parietal cortical stimulation. (A) Cell-density maps for c-

532 Fos-positive cells at three bregma levels $(+0.96,-3.60)$ in the sham operation group and the

533 experimental groups that received SEBS at 20,40, 60, or $100 \mathrm{~Hz}$ (left), together with the atlas

534 reference section (right). (B) Automated cell counts in four regions of interest, i.e., the motor cortex,

535 sensory cortex, striatum, and thalamus. Comparing stimulated groups with the sham group showed

536 that SEBS increased cFos activity. SEBS at $40 \mathrm{~Hz}$ achieved the highest increment in c-Fos in the

537 motor and sensory cortex and SEBS at $20 \mathrm{~Hz}$ showed the highest increment of c-Fos in the thalamus.

538 The error bars represent the standard error of the mean. ${ }^{*} p<0.05$ and $* * * p<0.001$ compared with the

539 other study groups, one-way analysis of variance with Bonferroni post-hoc test. Scale bar: C, $3 \mathrm{~mm}$.

540 EBS, electrical brain stimulation; n.s., not statistically significant 
542 Fig. 4. Histological confirmation of differential electrical stimulation and pattern of effect

543 during cortical stimulation of the sensory parietal cortex for 1 week. (A) Immunostaining of a rat

544 cortex with CaMKII and c-Fos antibodies. CaMKII and c-Fos double-positive cells indicate activated

545 excitatory neurons. (B) Immunostaining of a rat cortex with PV and c-Fos antibodies. PV and c-Fos

546 double-positive cells indicate activated inhibitory neurons. (C) Quantification of the activated neuron

547 ratio in the rat cortex in response to sinusoidal electrical stimulation at different frequencies, i.e., sham,

$54820 \mathrm{~Hz}, 40 \mathrm{~Hz}$, and $60 \mathrm{~Hz}$. (D, E) Quantification of the ratio of activated neurons from the mean value

549 for the rat cortex in the sham group in response to sinusoidal electrical stimulation at frequencies of

550 20, 40, and 60 Hz. (D) Comparison between CaMKII and PV at specific frequencies. (E) Comparison

551 between different frequencies according to specific regional cell type. The white arrow represents c-

552 Fos and CaMKII or c-Fos and PV double-positive cells. The error bars represent the standard error of

553 the mean. $* p<0.05$ and $* * * p<0.001$ compared with the control, one-way analysis of variance with

554 Bonferroni post-hoc test. Scale bars: B, $500 \mu \mathrm{m}$ and $50 \mu \mathrm{m}$. CaMKII, calmodulin-dependent protein

555 kinase II; PV, parvalbumin; n.s., not statistically significant

556 Fig. 5. Simulated current density distribution. The spatial distributions of current density induced

557 by $1 \mathrm{~V}$ stimulus amplitude are visualized at the surface of the brain (A) and the cross-section passing

558 the reference (B) and active (C) electrodes (following black dotted line shown in (A)) are shown.

559 Fig. 6. Map showing the relationship between firing frequency and sinusoidal stimulation. The

560 spatial distributions of the firing rate for the inhibitory (A) and excitatory (B) neuron model induced

561 by different stimulus amplitudes and stimulus frequencies are depicted. The firing rate is analyzed by

562 action potentials (APs) efficiency, which define percentage action potentials per stimulation pulse. An

563 APs efficiency value $>1$ indicates burst, a value of 1 indicate phase lock, and a value of $<1$ indicates

564 intermittent firing behavior. The blue contour lines represent the 1:1 phase-locked firing region.

565 Fig. 7. Simulated spatial distribution of firing rate. The spatial distributions of the firing rate

566 induced by a $1-\mathrm{V}$ stimulus amplitude are visualized on the surface of the brain for excitatory and 
bioRxiv preprint doi: https://doi.org/10.1101/855395; this version posted November 26,2019 . The copyright holder for this preprint (which was not certified by peer review) is the author/funder, who has granted bioRxiv a license to display the preprint in perpetuity. It is made available under aCC-BY-NC-ND 4.0 International license.

567 inhibitory neurons by increasing the stimulus frequency in steps of $20 \mathrm{~Hz}$. 


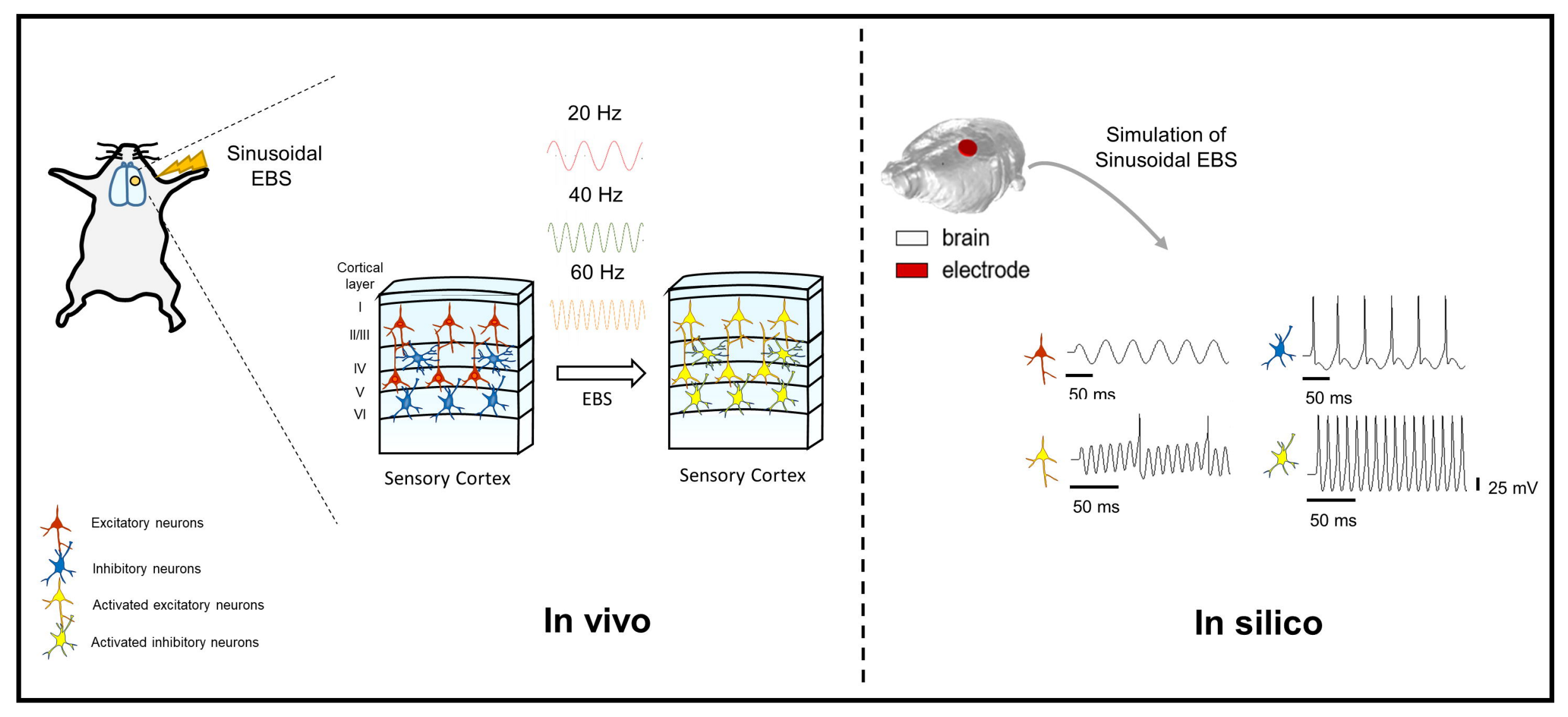


Figure 1. Schematic diagram of experimental schedule and example cell density heat map of c-Fos positive cells. (A) Experimental procedure of sinusoidal EBS in the SD rats. (B) Example image showed customized transfer function for Cell-density maps of c-Fos positive cells 


\section{Figure 1}

A

\section{Electrode Insertion}

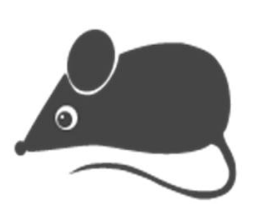

\begin{tabular}{c|c} 
& Postoperative Recovery \\
\hline &
\end{tabular}

Day 0
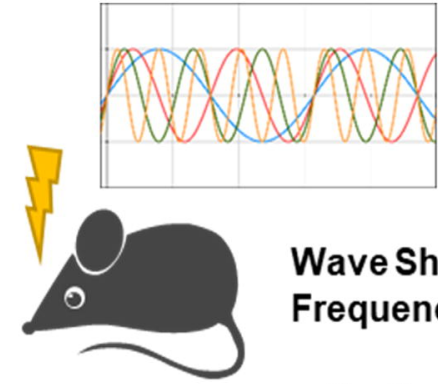

Wave Shape: Sine

Frequency(Hz) : 20, 40, 60, $100 \mathrm{~Hz}$

Cortical Stimulation

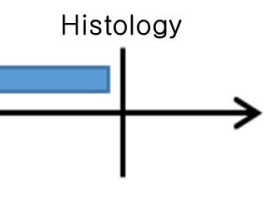

Day 7

Day 14

B

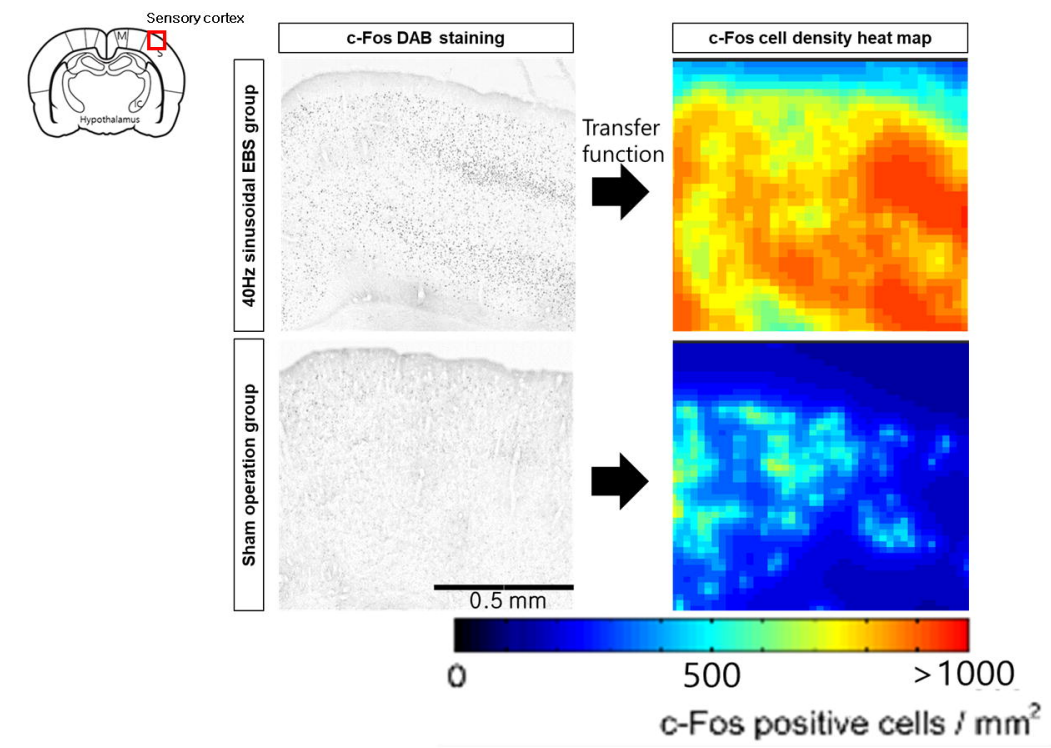


Figure 2. High-resolution CT based anatomically realistic rat head model. The anatomically realistic rat head model consisted of four layers of skin (a), skull (b), CSF (c), and brain (d). The electrode placement is placed in accordance to coordinates used in the experiment (e), and the cross-section (following black dotted line shown in (e)) passing the reference (f) and the active electrode $(\mathrm{g})$ are shown. 
Figure 2

(a)

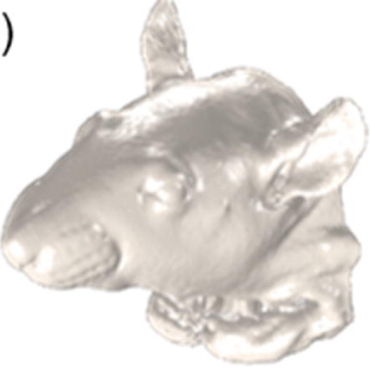

(e)

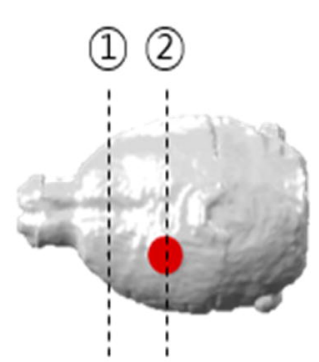

(b)

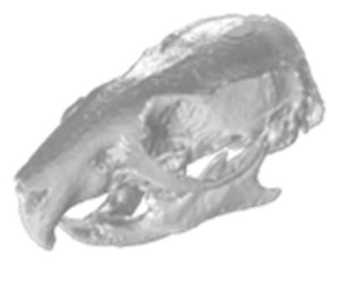

(f)

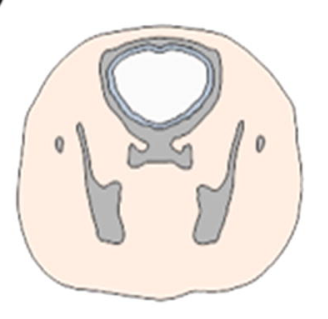

(c)

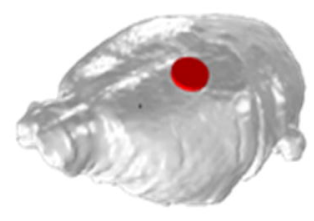

(g)

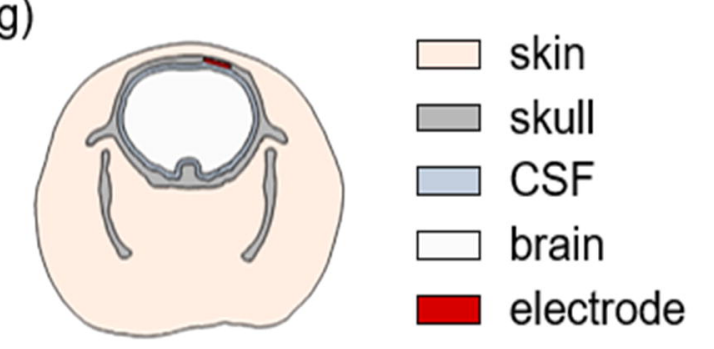

(d)

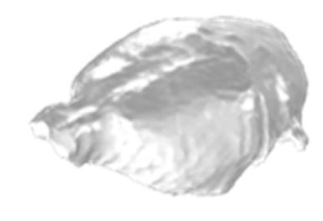

electrode 
Figure 3. Expression of c-Fos after sensory-parietal cortical stimulation. (A) Cell-density maps for c-Fos-positive cells at three bregma levels $(+0.96,-3.60)$ in the sham operation group and the experimental groups that received SEBS at 20,40,60, or $100 \mathrm{~Hz}$ (left), together with the atlas reference section (right). (B) Automated cell counts in four regions of interest, i.e., the motor cortex, sensory cortex, striatum, and thalamus. Comparing stimulated groups with the sham group showed that SEBS increased cFos activity. SEBS at $40 \mathrm{~Hz}$ achieved the highest increment in cFos in the motor and sensory cortex and SEBS at $20 \mathrm{~Hz}$ showed the highest increment of c-Fos in the thalamus. The error bars represent the standard error of the mean. ${ }^{*} p<0.05$ and ${ }^{*} * * p<0.001$ compared with the other study groups, one-way analysis of variance with Bonferroni post-hoc test. Scale bar: C, 3 mm. EBS, electrical brain stimulation; n.s., not statistically significant 
Figure 3

A

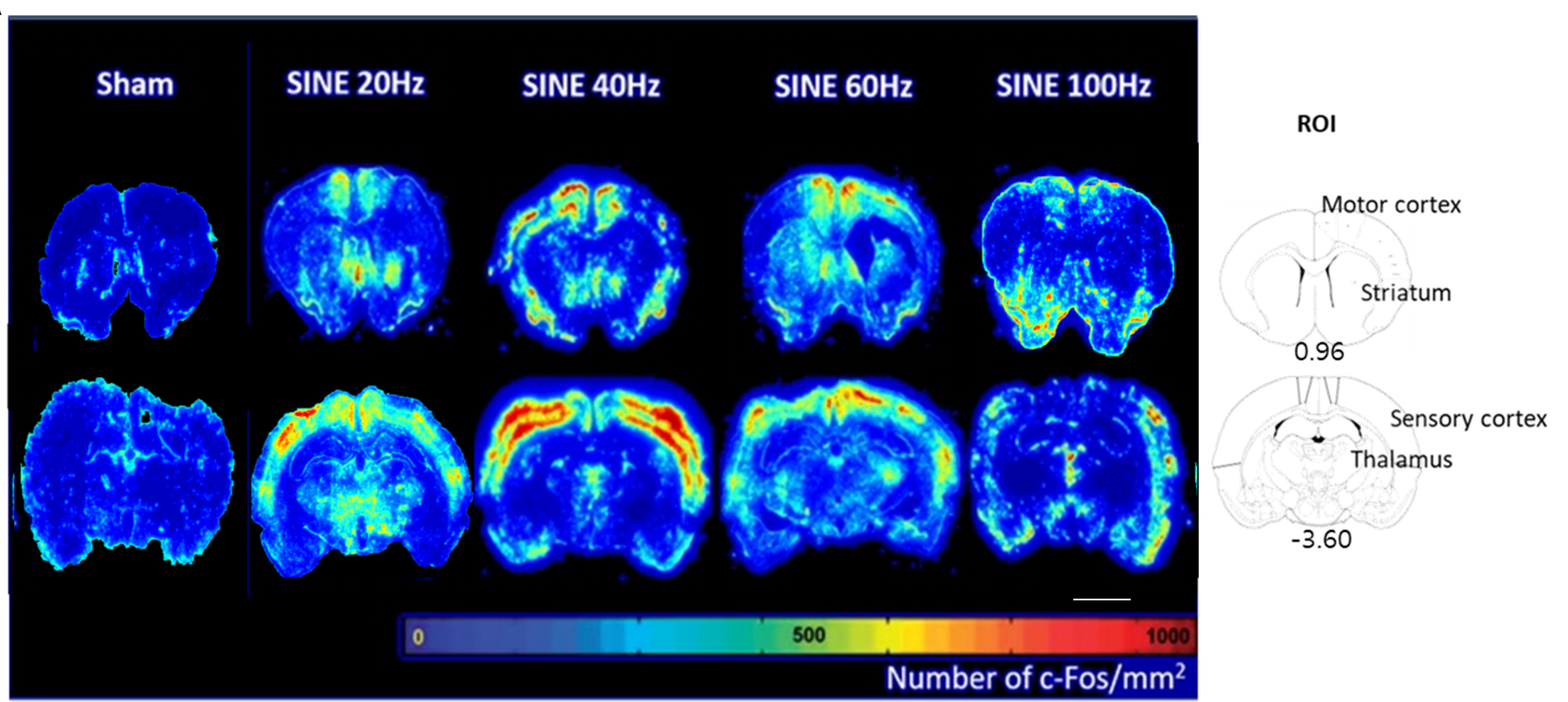



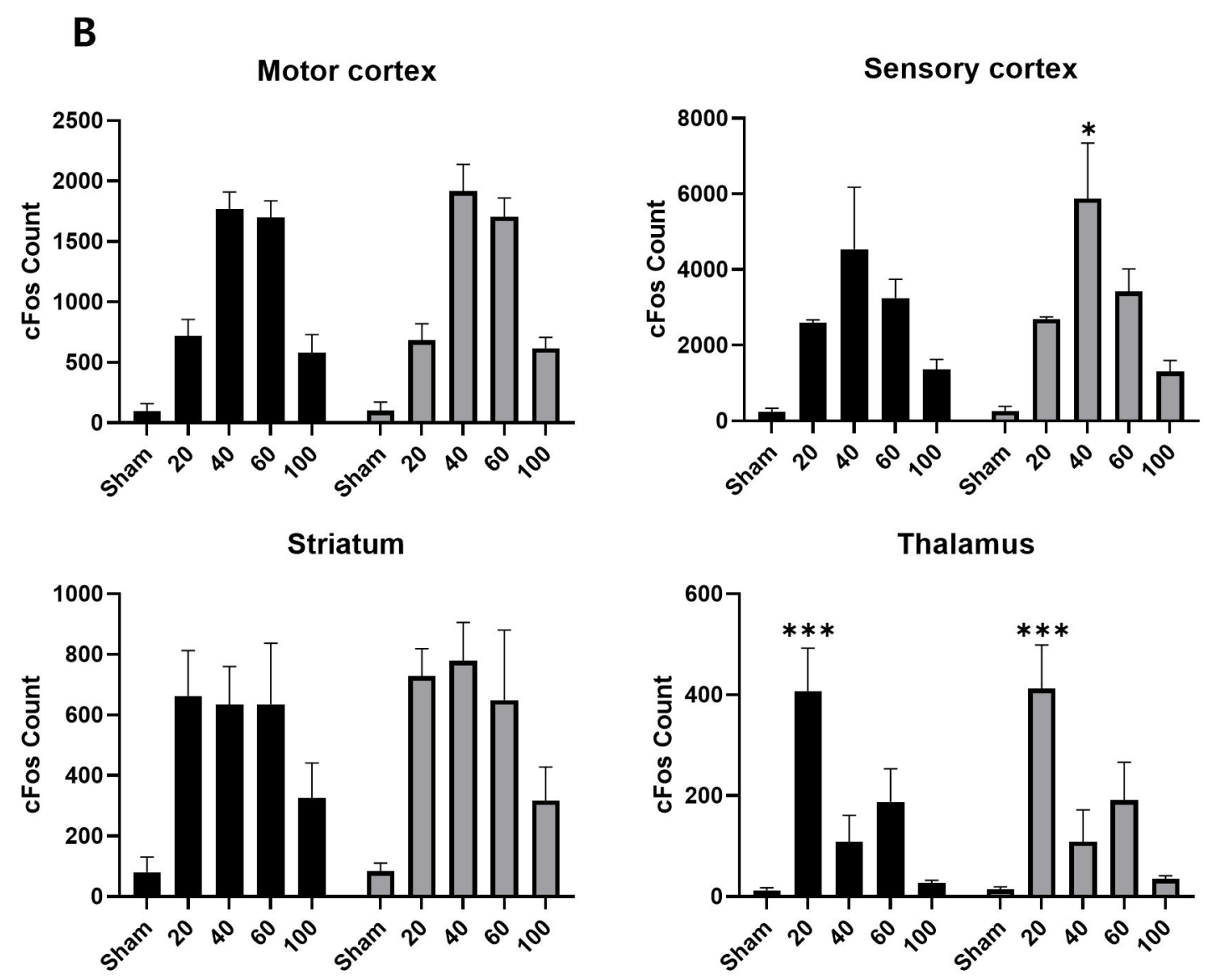
Figure. 4. Histological confirmation of differential electrical stimulation and the pattern of effect during one week

of cortical stimulation in sensory-parietal cortex. (A) Immunostaining of a rat cortex with CaMKII and c-Fos antibodies. CaMKII and c-Fos double-positive cells indicate activated excitatory neurons. (B) Immunostaining of a rat cortex with PV and c-Fos antibodies. PV and c-Fos double-positive cells indicate activated inhibitory neurons. (C) Quantification of the activated neuron ratio in the rat cortex in response to sinusoidal electrical stimulation at different frequencies, i.e., sham, $20 \mathrm{~Hz}, 40 \mathrm{~Hz}$, and $60 \mathrm{~Hz}$. (D, E) Quantification of the ratio of activated neurons from the mean value for the rat cortex in the sham group in response to sinusoidal electrical stimulation at frequencies of 20, 40, and $60 \mathrm{~Hz}$. (D) Comparison between CaMKII and PV at specific frequencies. (E) Comparison between different frequencies according to specific regional cell type. The white arrow represents c-Fos and CaMKII or c-Fos and PV double-positive cells. The error bars represent the standard error of the mean. ${ }^{*} \mathrm{p}<0.05$ and ${ }^{* * *} \mathrm{p}<0.001$ compared with the control, one-way analysis of variance with Bonferroni post-hoc test. Scale bars: B, $500 \mu \mathrm{m}$ and $50 \mu \mathrm{m}$. CaMKII, calmodulin-dependent protein kinase II;

PV, parvalbumin; n.s., not statistically significant 
Figure 4

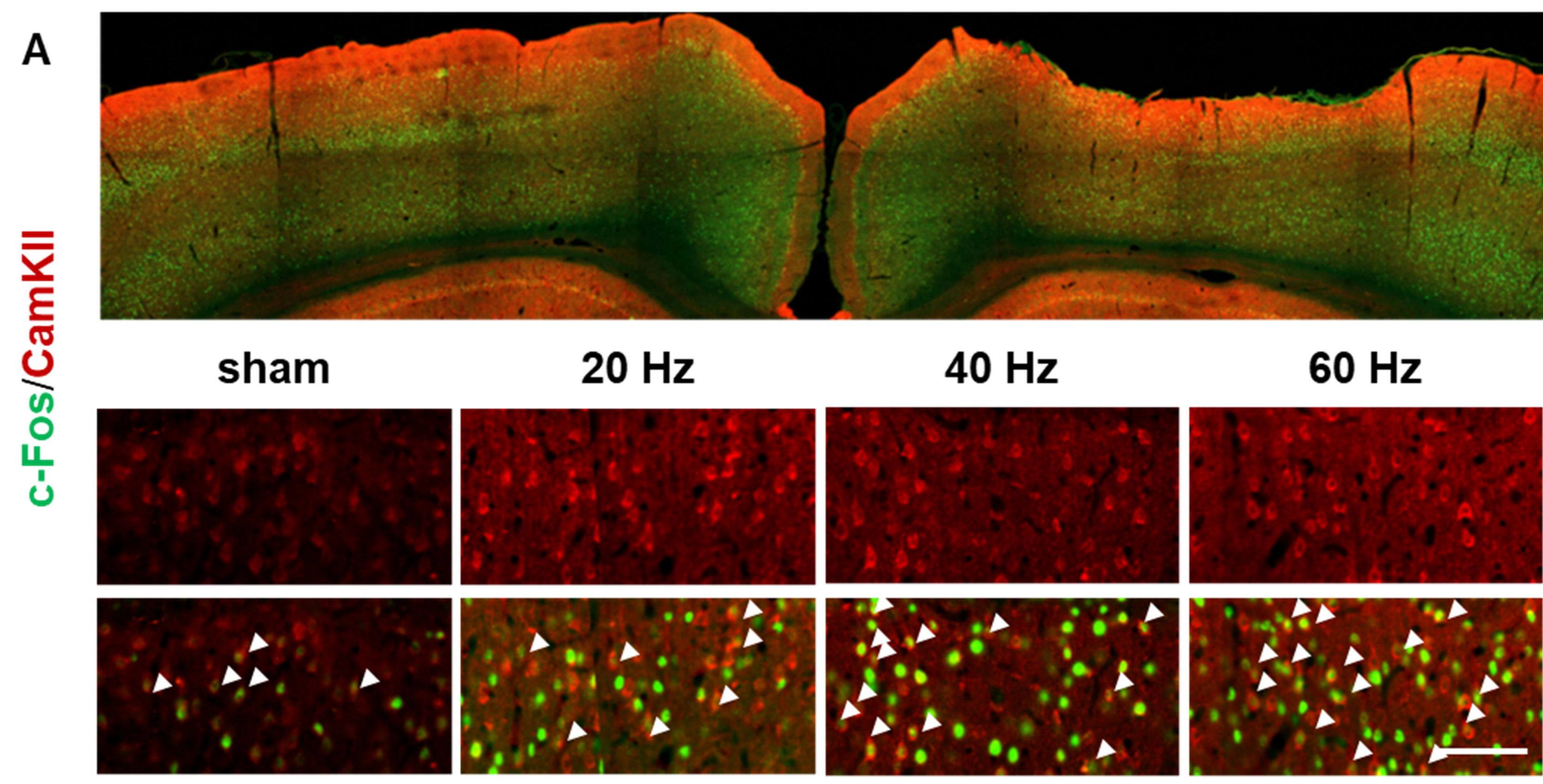


B

sham

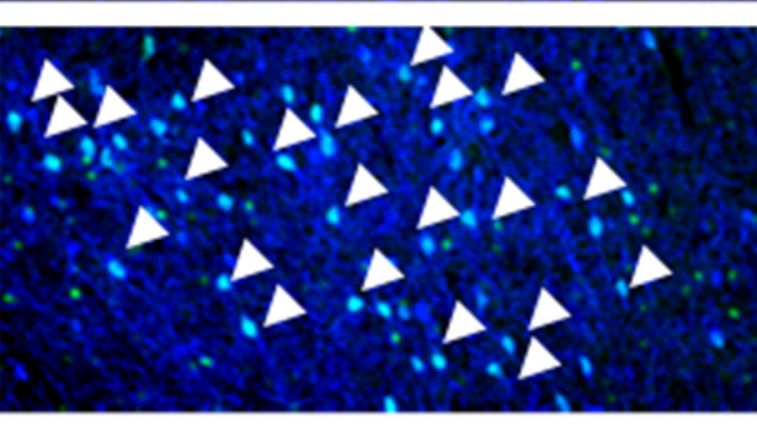

$20 \mathrm{~Hz}$

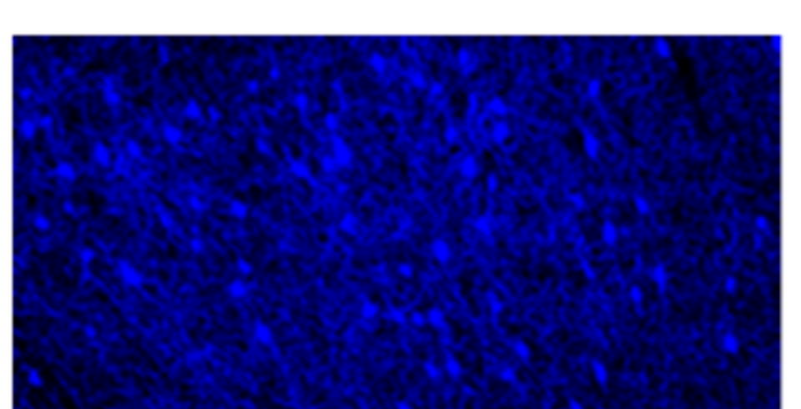

$40 \mathrm{~Hz}$

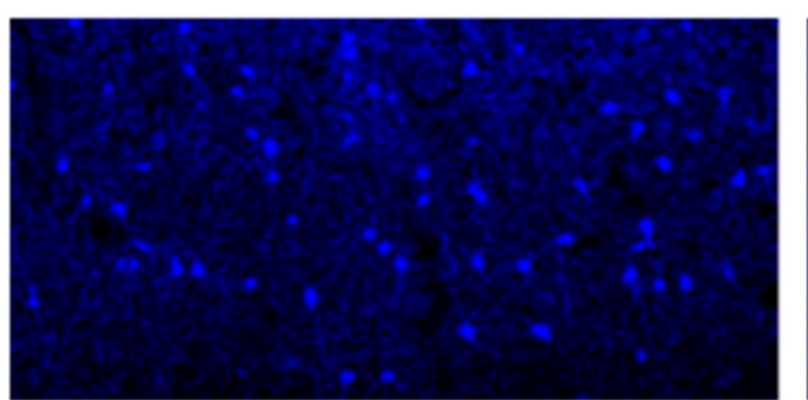

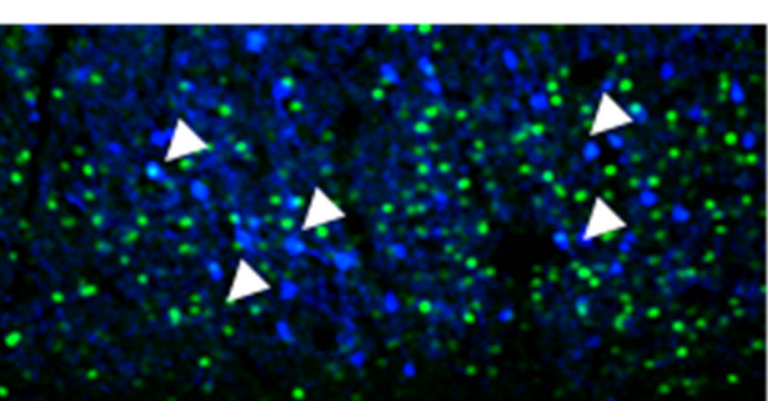
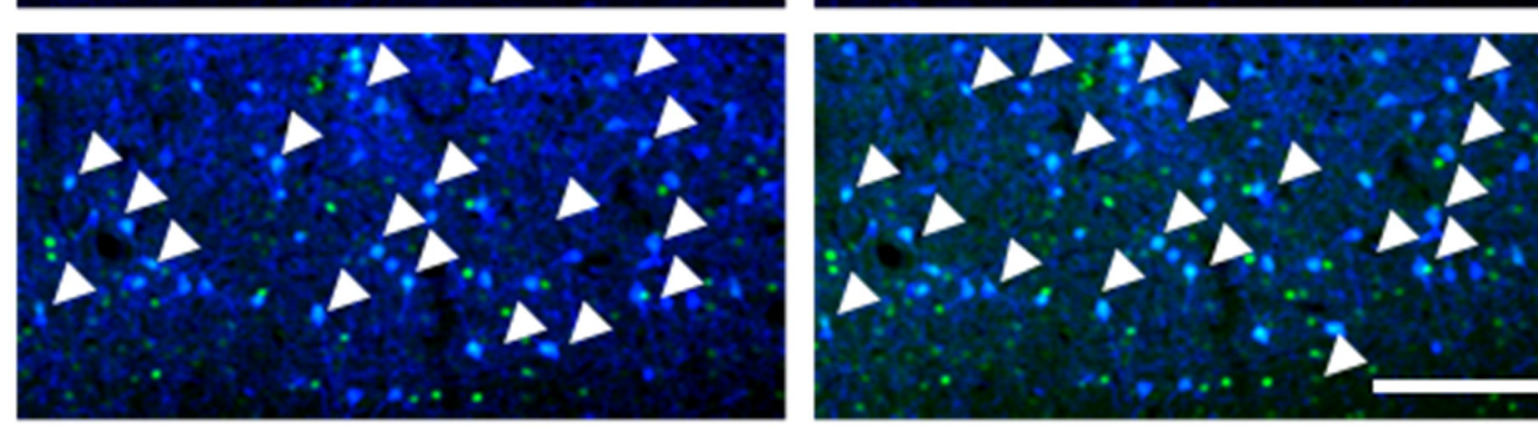


\section{Figure 5}

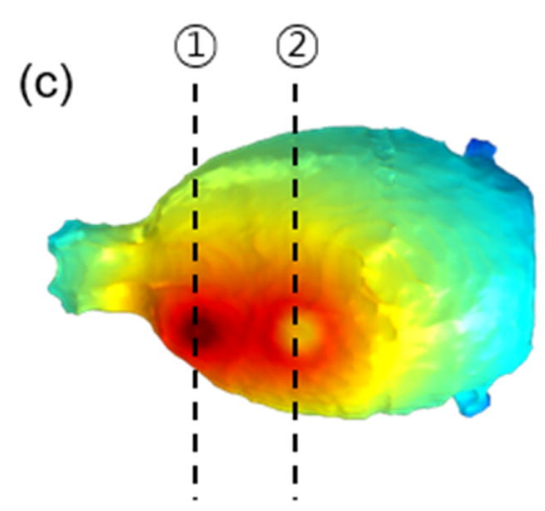

(d)

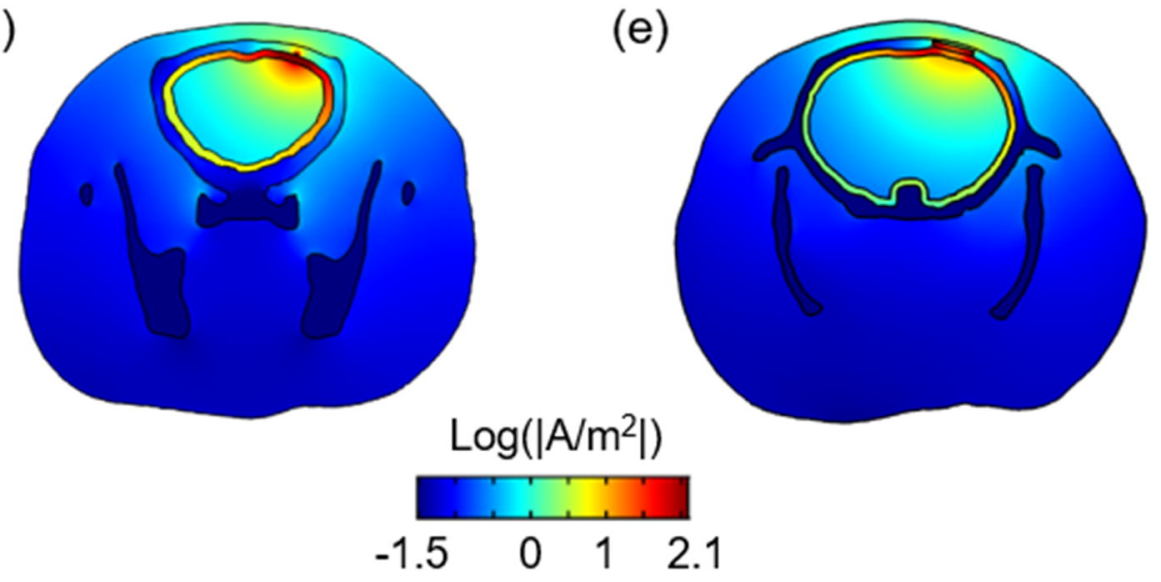

Figure 5. Simulated current density distribution. The spatial distributions of current density induced by $1 \mathrm{~V}$ stimulus amplitude are visualized at the surface of the brain (c) and the crosssection passing the reference (d) and active (e) electrodes (following black dotted line shown in (c)) are shown. 


\section{Figure 6}

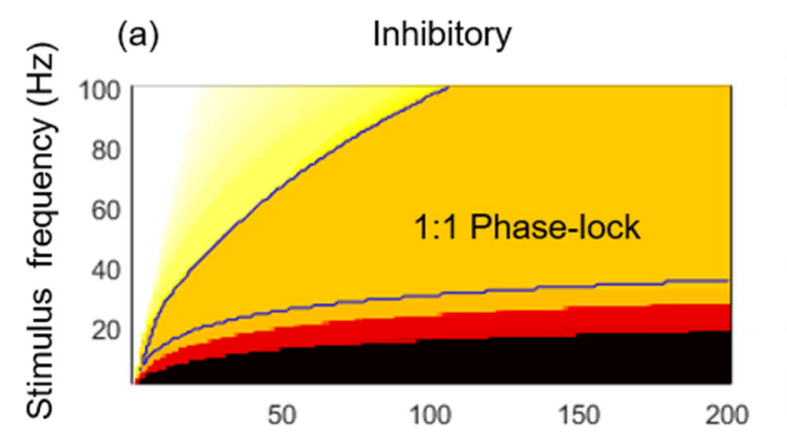

Stimulus amplitude $(n A)$

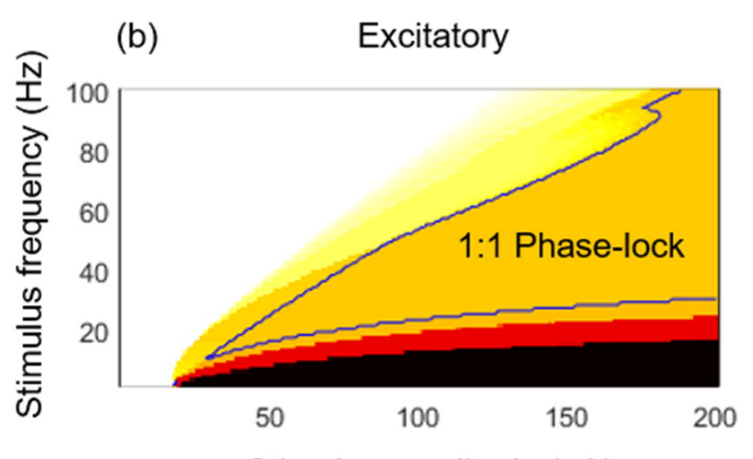

Stimulus amplitude $(\mathrm{nA})$

Figure 6. Relation map between firing frequency and sinusoidal stimulation. The spatial distributions of firing rate of inhibitory (a) and excitatory (b) neuron model induced by different stimulus amplitude and stimulus frequency are depicted. The firing rate is analyzed by action potentials (APs) efficiency and the blue contour lines represent 1:1 phase locked firing region. 


\section{Figure 7}

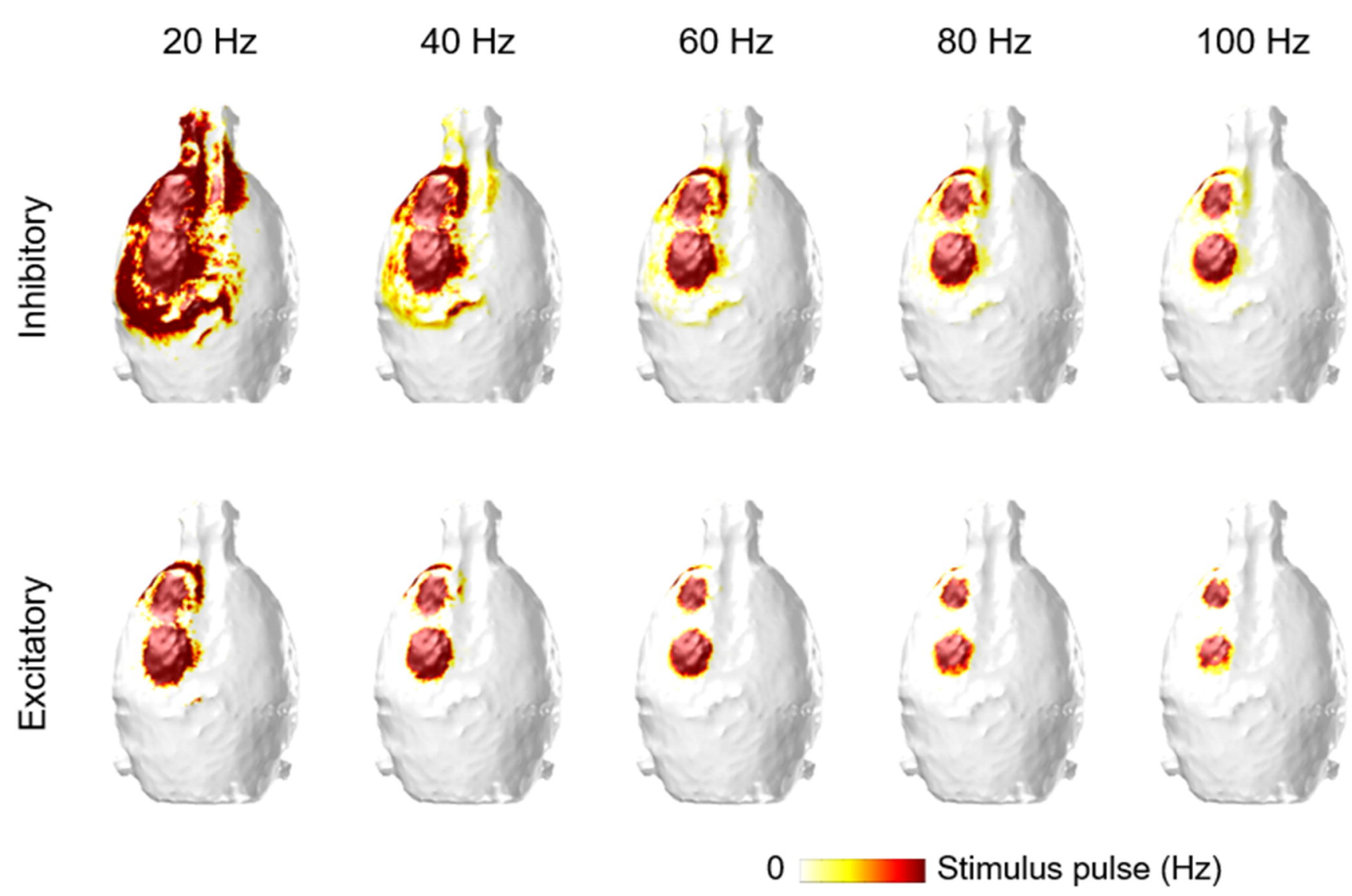

Fig. 7. Simulated spatial distribution of firing rate. The spatial distributions of firing rate induced by $1 \mathrm{~V}$ stimulus amplitude are visualized at the surface of the brain for excitatory and inhibitory neurons by increasing stimulus frequency in steps of $20 \mathrm{~Hz}$. 


\section{Supplementary Figure S1}

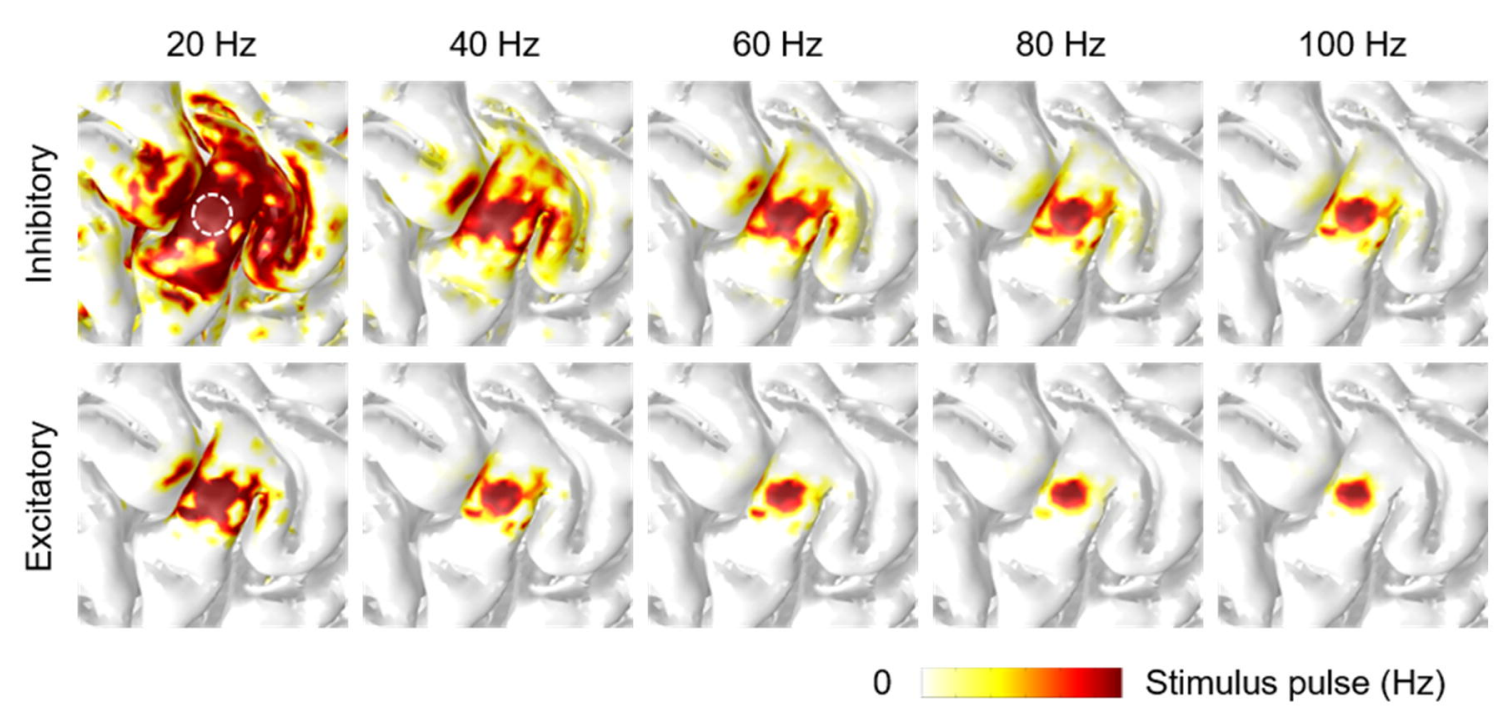

Figure S1. Simulated spatial distribution of firing rate subject to the human model.

Simulated spatial distribution of firing rate subject to the human model. The spatial distribution of firing rate induced by $1 \mathrm{~V}$ stimulus amplitude are depicted at the cortical surface for excitatory and inhibitory neurons, by increasing stimulus frequency in steps of $20 \mathrm{~Hz}$. The active electrode targeting sensory cortex is marked by while-colored and dotted line in $20 \mathrm{~Hz}$ stimulus-induced inhibitory neuronal responses. 


\section{Supplementary Figure S2}

Figure S2. Investigation of activated neuronal cell types at the cortical layer localization. (A) Representative image of CamKII, PV and c-Fos immunostaining at rat cortex. (B-D) Distribution analysis of activated neurons in the rat cortex. Error bars represent S.E.M. ${ }^{*} p<0.05$ compared with sham control, two way analysis of variance on ranks with Dunn's method.

Scale bars: B, $200 \mu \mathrm{m}$

A

C-Fos/CamKIIIPV $B$
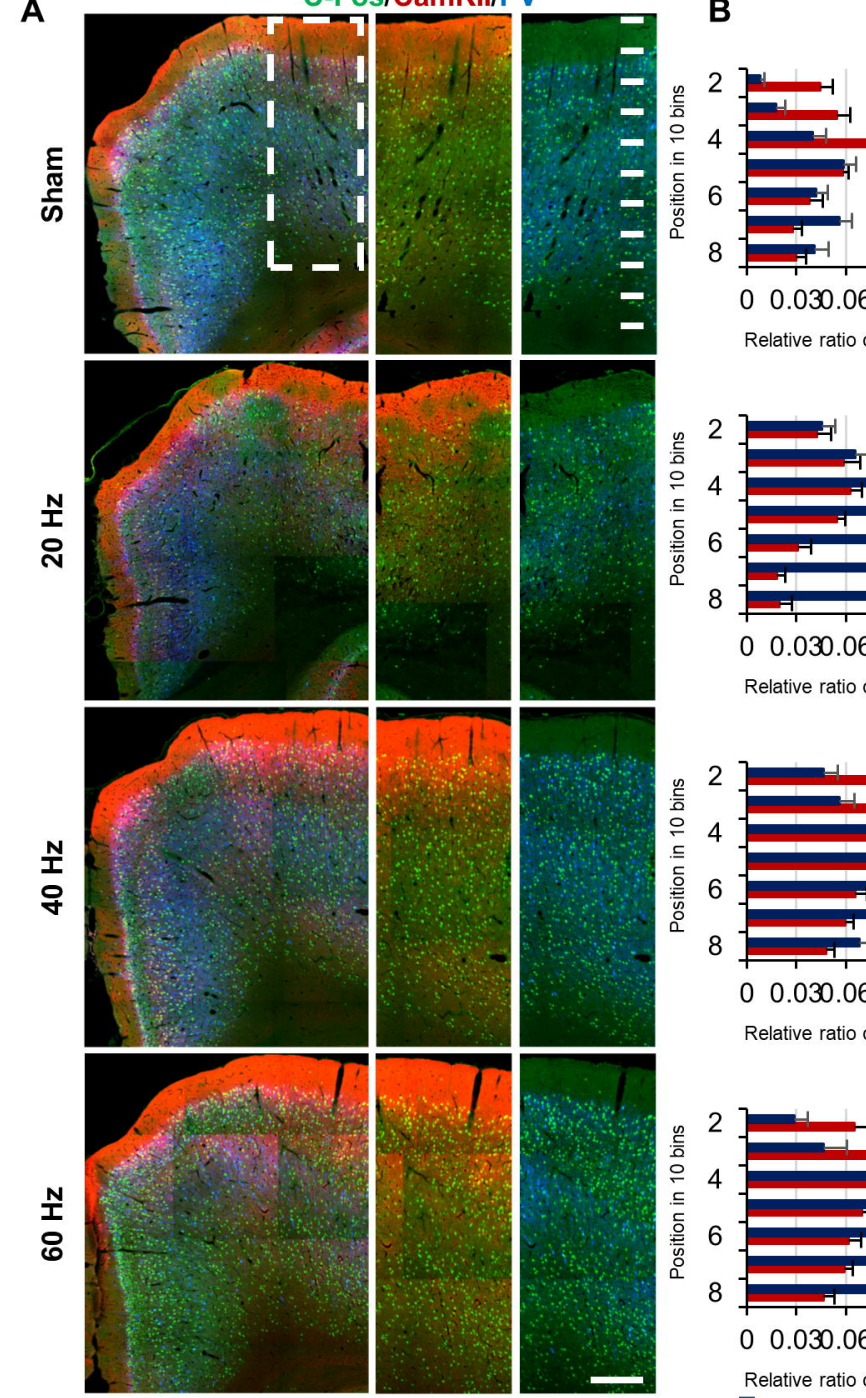

$+1$

0 0.030.060.090.120.15 Relative ratio of activated cells
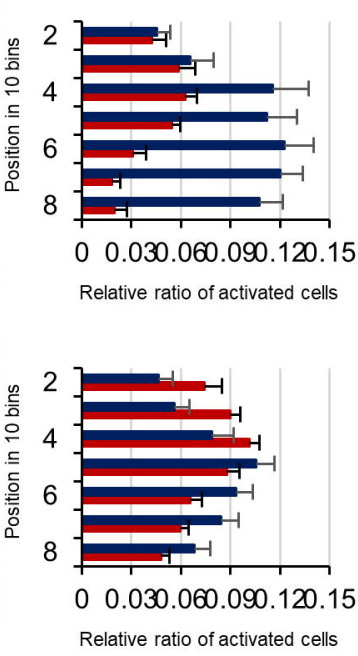

Relative ratio of activated cells

Relative ratio of activated cells
- \% of cFos $+\mathrm{PV}^{+}+$trotal PV
- of cells
of cFos

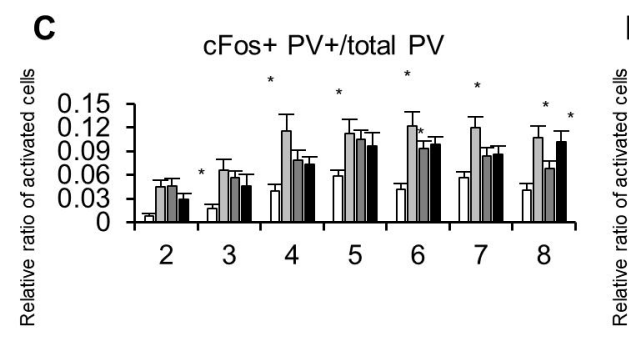

D

cFos+CamKII+/total CamKII

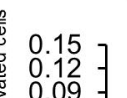

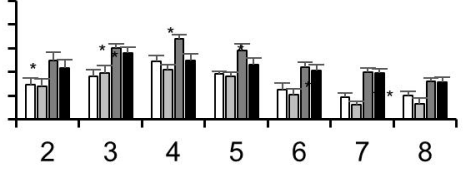

口sham 


\section{Supplementary Table 1}

\begin{tabular}{|c|c|c|c|c|c|c|c|c|c|c|}
\hline \multirow[b]{2}{*}{ unit: cells } & \multicolumn{5}{|c|}{ Ipsilateral } & \multicolumn{5}{|c|}{ Contralateral } \\
\hline & Sham $(n=5)$ & $20 \mathrm{~Hz}(\mathrm{n}=4)$ & $40 \mathrm{~Hz}(n=7)$ & $60 \mathrm{~Hz}(\mathrm{n}=4)$ & $100 \mathrm{~Hz}(\mathrm{n}=7)$ & Sham $(n=5)$ & $20 \mathrm{~Hz}(n=4)$ & $40 \mathrm{~Hz}(n=7)$ & $60 \mathrm{~Hz}(n=4)$ & $100 \mathrm{~Hz}(\mathrm{n}=7)$ \\
\hline $\begin{array}{l}\text { Motor } \\
\text { cortex }\end{array}$ & $98.4 \pm 24.14$ & $717 \pm 59.85$ & $\begin{array}{c}1768.29 \pm \\
49.61\end{array}$ & $1697 \pm 60.72$ & $\begin{array}{c}577.86 \pm \\
53.61\end{array}$ & $101.8 \pm 28.06$ & $683.75 \pm 59.20$ & $1916.57 \pm 78.04$ & $1706.25 \pm 66.38$ & $616 \pm 32.10$ \\
\hline $\begin{array}{c}\text { Sensory } \\
\text { cortex }\end{array}$ & $245 \pm 82.61$ & $\begin{array}{c}2595.25 \\
\pm 33.78\end{array}$ & $4539 \pm 573.03$ & $\begin{array}{c}3248.5 \pm \\
214.85\end{array}$ & $\begin{array}{c}1554.14 \pm \\
129.79\end{array}$ & $264.4 \pm 47.28$ & $2687 \pm 27.17$ & $5877.14 \pm 513.63$ & $3423.75 \pm 256.16$ & $1530.29 \pm 124.85$ \\
\hline Striatum & $80.2 \pm 44.86$ & $662.5 \pm 65.23$ & $\begin{array}{c}634.43 \pm \\
43.77\end{array}$ & $635 \pm 87.39$ & $\begin{array}{c}326.29 \pm \\
40.27\end{array}$ & $83.6 \pm 10.59$ & $727.5 \pm 39.58$ & $779 \pm 44.31$ & $648.75 \pm 100.24$ & $316.71 \pm 39.04$ \\
\hline Thalamus & $12.4 \pm 4.45$ & $\begin{array}{c}407.25 \pm \\
36.79\end{array}$ & $\begin{array}{c}108.29 \pm \\
18.31\end{array}$ & $\begin{array}{c}187.75 \pm \\
28.31\end{array}$ & $26.86 \pm 1.94$ & $14 \pm 2.12$ & $412 \pm 37.60$ & $108.57 \pm 22.05$ & $191.25 \pm 32.55$ & $35 \pm 2.26$ \\
\hline
\end{tabular}

Table S1. The number of c-Fos expression after sensory-parietal cortical stimulation.

Automated cell counts of sham, 20,40,60,100Hz group in four different regions of interest: motor cortex, sensory cortex, striatum, thalamus. Comparing stimulated groups with sham operation group showed that sinusoidal EBS increased c-Fos activity. 